\title{
Ultrathin Epitaxial Silicon Solar Cells with Inverted Nanopyramid Arrays for Efficient Light Trapping
}

\author{
Alexandre Gaucher, ${ }^{\dagger}$ Andrea Cattoni, ${ }^{\dagger}$ Christophe Dupuis, ${ }^{\dagger}$ Wanghua Chen, ${ }^{\dagger}$ Romain Cariou, ${ }^{\ddagger}$ \\ Martin Foldyna, ${ }^{\ddagger}$ Loïc Lalouat, ${ }^{\S}$ Emmanuel Drouard, ${ }^{\S}$ Christian Seassal, ${ }^{\S}$ Pere Roca i Cabarrocas, ${ }^{\ddagger}$ \\ and Stéphane Collin*,
}

\author{
${ }^{\dagger}$ Centre de Nanosciences et de Nanotechnologies, CNRS, University Paris Sud, Université Paris-Saclay, C2N-Marcoussis, 91460 \\ Marcoussis, France \\ ${ }^{\ddagger}$ LPICM, CNRS, Ecole Polytechnique, University Paris-Saclay, 91128 Palaiseau, France \\ ${ }^{\S}$ Institut des Nanotechnologies de Lyon (INL), Université de Lyon, UMR5270, CNRS-INSA-ECL-UCBL, Ecole Centrale de Lyon, \\ Ecully 69134, France
}

\begin{abstract}
Ultrathin c-Si solar cells have the potential to drastically reduce costs by saving raw material while maintaining good efficiencies thanks to the excellent quality of monocrystalline silicon. However, efficient light trapping strategies must be implemented to achieve high short-circuit currents. We report on the fabrication of both planar and patterned ultrathin c-Si solar cells on glass using low temperature $\left(\mathrm{T}<275^{\circ} \mathrm{C}\right)$, low-cost, and scalable techniques. Epitaxial c-Si layers are grown by PECVD at $160{ }^{\circ} \mathrm{C}$ and transferred on a glass substrate by anodic bonding and mechanical cleavage. A silver back mirror is combined with a front texturation based on an inverted nanopyramid array fabricated by nanoimprint lithography and wet etching. We demonstrate a short-circuit current density of $25.3 \mathrm{~mA} / \mathrm{cm} 2$ for an equivalent thickness of only $2.75 \mu \mathrm{m}$. External quantum efficiency (EQE) measurements are in very good agreement with FDTD simulations. We infer an optical path enhancement of 10 in the long wavelength range. A simple propagation model reveals that the low photon escape probability of $25 \%$ is the key factor in the light trapping mechanism. The main limitations of our current technology and the potential efficiencies achievable with contact optimization are discussed.
\end{abstract}

KEYWORDS: solar cells, crystalline silicon, low-temperature epitaxy, light trapping, nanoimprint lithography

S licon is the dominant technology for photovoltaic solar cells. Efficiencies of $25.6 \%$ have been achieved at the cell level, ${ }^{1}$ which is close to the $29.4 \%$ efficiency limit for crystalline silicon solar cells. ${ }^{2}$ One of the main challenges facing current silicon technologies is the reduction of costs at the module and cell levels. A rationale way is to reduce the consumption of purified crystalline silicon. Indeed, the thickness of state-of-theart solar cells $(100-200 \mu \mathrm{m})$ is far from the theoretical limits and could be reduced by more than 1 order of magnitude with efficient light-trapping. ${ }^{3}$ In wafer-based technologies, however, reducing the wafer thickness cannot be pursued below $100 \mu \mathrm{m}$ thick absorbers because of handling issues and important kerf losses. In this sense, thin-film silicon-based technologies are a promising way to further reduce the silicon consumption. On the one hand, amorphous silicon (a-Si:H) and microcrystalline silicon $(\mu \mathrm{c}-\mathrm{Si}: \mathrm{H})$ thin films benefit from a high absorptivity, but suffer from low overall efficiencies as compared to wafer-based crystalline solar cells ${ }^{1}$ due to poor transport properties. They can be improved by rapid thermal crystallization and lead to thin crystalline silicon on glass (CSG) solar cells. Although not monocrystalline, record modules have reached efficiencies of $10.5 \%$ and $J_{\mathrm{sc}}=29.7 \mathrm{~mA} / \mathrm{cm}^{2}$ with silicon thicknesses below 2 $\mu \mathrm{m} .^{4-6}$ On the other hand, the emerging field of thin film crystalline silicon (c-Si) solar cells has the potential for reducing the silicon consumption while maintaining high efficiencies thanks to the excellent quality of monocrystalline silicon.

Recent numerical studies have shown that state-of-the-art thick c-Si solar cell efficiencies could be achieved using only a few-micrometer-thick c-Si layers ${ }^{7,8}$ when efficient light trapping strategies are put in place. Several techniques have been used to produce thin crystalline silicon layers such as epitaxial growth on porous silicon and transfer to a foreign substrate, ${ }^{9,10}$ exfoliation of a c-Si foil from a commercial wafer ${ }^{11}$ or "epi-free" process based on recrystallization of amorphous material deposited on a foreign substrate. ${ }^{12}$ Different light trapping strategies have been described in the literature such as the use 
of photonic crystals, ${ }^{13}$ diffraction gratings, ${ }^{14-16}$ plasmonic scatterers, $^{17,18}$ Mie resonators, ${ }^{19}$ or aperiodic structures. ${ }^{20}$ Numerical issues can arise from absence of periodicity or from the different length scales (nanoscale texturation and thicknesses up to several tens of microns). In such cases, propagation matrix models have been proposed recently as an alternative to conventional electromagnetic numerical methods. $^{21,22}$ Crystalline silicon solar cells with absorbing layer thickness of $43 \mu \mathrm{m}$ have shown very promising results with efficiencies of $19 \%{ }^{23}$ and $20.6 \%,{ }^{24}$ respectively. Several authors have focused on further reducing the absorber thickness below $10 \mu \mathrm{m} .{ }^{25-29}$ Interestingly, best short-circuit currents have been reported using wavelength scale structuration such as nanopyramids $^{29}$ or nanocones. ${ }^{25,27}$ Branham et al. ${ }^{29}$ recently reported an efficiency of $15.7 \%$ for a $10 \mu \mathrm{m}$ thick crystalline silicon absorbing layer. However, as for refs., ${ }^{25-29}$ commercial silicon on insulator (SOI) wafers were etched chemically to provide ultrathin silicon layer. Moreover, expensive and high temperature processes were used in these proof-of-concept studies.

The present work aims at producing ultrathin ( 2 to $10 \mu \mathrm{m}$ thick) crystalline silicon solar cells with low cost, low temperature, and scalable processes. It focuses on the fabrication of ultrathin c-Si layers transferred on glass and on the implementation of efficient light-trapping techniques to increase the short-circuit current. In particular, we chose an inverted pyramid array with a wavelength-scale pitch on the front side of the cells, combined with a silver back mirror. In the following, we describe the fabrication process and device characterization. A record short-circuit current density of $J_{\mathrm{sc}}=$ $25.3 \mathrm{~mA} / \mathrm{cm}^{2}$ is measured for $3 \mu \mathrm{m}$ thick monocrystalline solar cells. We then provide an in-depth optical analysis based on external quantum efficiency (EQE) measurements and numerical FDTD calculations. A simple propagation model is also used in order to reveal the key factors of the light-trapping mechanism: the high optical path enhancement of 10 is mainly due to the back mirror and the low photon escape probability (25\%). We finally discuss the main limitations of our current technology and the potential efficiencies achievable with further optimizations.

The $3 \mu \mathrm{m}$ thick c-Si epitaxial layers were grown by plasma enhanced chemical vapor deposition (PECVD) at low temperature $^{30,31}\left(T<200{ }^{\circ} \mathrm{C}\right)$ on 4 in. wafers (Figure 1a). The final structure of our cells is based on the heterojunction with intrinsic thin layer (HIT) design (Figure 1d). We use doped p-type a-Si:H $(14 \mathrm{~nm})$ and n-type $\mu \mathrm{c}-\mathrm{SiO}_{x}: \mathrm{H}(58 \mathrm{~nm})$ layers to form the top and back passivation layers, respectively. The $\mu \mathrm{c}-\mathrm{SiO}_{x}: \mathrm{H}$ layer is deposited by PECVD in the same run as the epilayer growth. This ensures a good interface and excellent passivation properties. In order to improve the absorbance in the transferred solar cell, a $\mathrm{ZnO} / \mathrm{Ag}(80 \mathrm{~nm} / 200 \mathrm{~nm})$ back mirror is added to the structure before the transfer process. The stack is bonded to a host glass substrate using anodic bonding at low temperature $\left(T=200{ }^{\circ} \mathrm{C}\right)$. A porous layer between the substrate and the epitaxial silicon layer, formed in situ by PECVD treatments, ${ }^{32,33}$ is used as a cleavage plane to mechanically remove the silicon substrate, as depicted in Figure $1 \mathrm{~b}$. The silicon substrate can be then reused to grow another epitaxial silicon layer, drastically reducing the cost of epitaxial silicon used in this technology. To fabricate planar c-Si solar cells on glass, the stack is directly completed with the deposition of the second passivation layer, an indium tin oxide (ITO, $80 \mathrm{~nm}$ ) layer as the transparent conductive oxide (TCO)

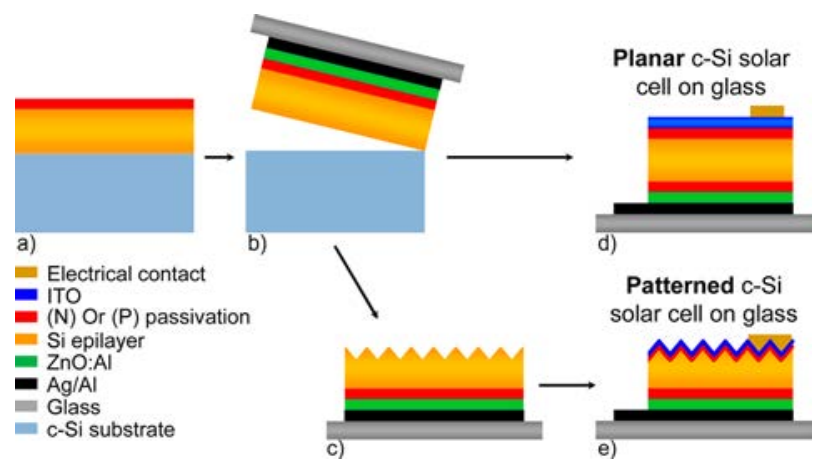

Figure 1. Process flow describing the fabrication of planar and nanopatterned c-Si solar cells on glass showing (a) the back passivation layer on top of the $\mathrm{c}$-Si epilayer after low temperature PECVD growth, (b) the bonding and transfer of the stack ( $\mathrm{Al} / \mathrm{Ag} /$ $\mathrm{ZnO}: \mathrm{Al} /(\mathrm{N})$-passivation/c-Si epilayer) to the glass substrate, (c) the structuration of the top surface of the epilayer with inverted nanopyramids, (d), (e) the final structure of a planar (d) and patterned (e) c-Si solar cell on glass.

and a top metallic contact (Figure 1d). The back mirror also acts as the metallic back electrode, and it is contacted via dry etching through the entire epilayer. The process is achieved with sample sizes of $1-2 \mathrm{~cm}^{2}$, and the final devices are circular cells with a diameter of $1 \mathrm{~mm}$.

In case of nanopatterned solar cells, an intermediate step is introduced to pattern inverted nanopyramids on the top surface of the epilayer (Figure 1c). The use of such an array has three main advantages: when properly designed, (i) it provides a graded index between the air and the cell that results in an efficient antireflection effect; (ii) it induces diffraction of light in the epilayer thus increasing the light path; and (iii) finally, it consumes less active material than the wafer etching without mask normally used to obtain random pyramids. Inverted pyramids are fabricated by wet chemical etching, which is less electrically harmful and cheaper than dry etching techniques. ${ }^{34}$ By using finite-difference time-domain (FDTD) calculations, we have identified a pitch of $800 \mathrm{~nm}$ as the most suitable to maximize light trapping in the c-Si epilayer while ensuring a broadband ARC property. Soft UV nanoimprint lithography (soft UV NIL) was used to fabricate a Cr etching mask, as this technique is advantageous to fabricate low cost nanostructures over large surface areas. In particular, soft UV NIL uses a poly(dimethylsiloxane) (PDMS) flexible stamp which is replicated from a single and expensive silicon master fabricated by electron beam lithography and dry etching. We used a technology based on hard-PDMS/PDMS in order to improve the resolution and reduce the line edge roughness. ${ }^{35,36}$ The inexpensive stamp can be used up to 500 times and is replicated again from the silicon master when deteriorated. ${ }^{37}$ Finally, the fabrication process is done keeping the temperature below 275 ${ }^{\circ} \mathrm{C}$ and the overall thermal budget is low. Inverted pyramids are obtained by $\mathrm{KOH}$ etching through the chromium hard mask (Figure 1e).

Scanning electron microscope (SEM) images of the resulting inverted pyramid arrays are presented in Figure $2 \mathrm{a}$ and $2 \mathrm{~b}$. They were fabricated over surface areas up to $1 \mathrm{~cm}^{2}$. Figure $2 \mathrm{c}$ shows a SEM cross section of the complete stack of a patterned solar cell transferred on a glass substrate. The different layers are highlighted with different colors. The top passivation layer and the ITO layer are conformally deposited on the inverted 

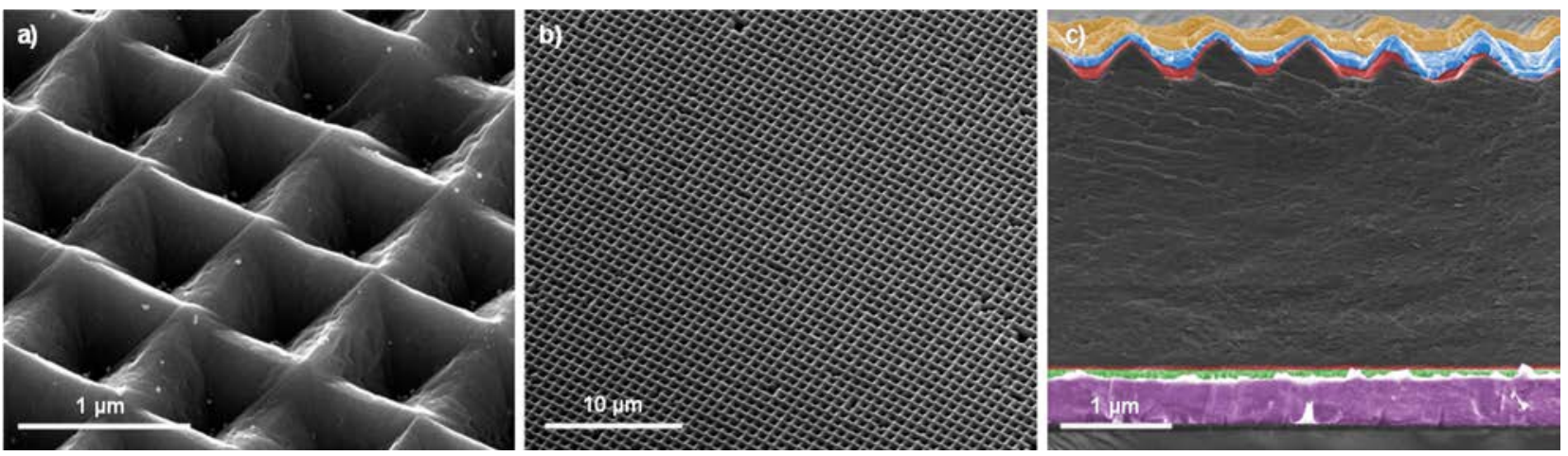

Figure 2. (a), (b) SEM images of the inverted pyramid array resulting from soft UV nanoimprint lithography and KOH etching. (c) Colorized SEM image of the cross-section of the complete stack of a patterned solar cell on glass. From bottom to top: glass substrate, metallic back contact/mirror (purple), $\mathrm{ZnO}: \mathrm{Al}$ optical spacer (green), $\mathrm{n}$-type $\mu \mathrm{c}-\mathrm{SiO}_{x}: \mathrm{H}$ passivation layer (red), c-Si epilayer, p-type a-Si:H passivation layer (red), ITO (blue), top metallic contact (yellow).

pyramids, which ensures an excellent passivation of the c-Si epilayer and a proper charge collection.

Planar and patterned solar cells on glass were fabricated from the same parent $\mathrm{c}-\mathrm{Si}$ epilayer with an initial thickness $d=3 \mu \mathrm{m}$. The equivalent thickness of patterned solar cells is defined as the thickness of a planar solar cell with the same volume of active c-Si material, which resulted in a thickness $d_{\mathrm{eq}}=2.75 \mu \mathrm{m}$ after etching of the inverted pyramids. Figure 3 shows the

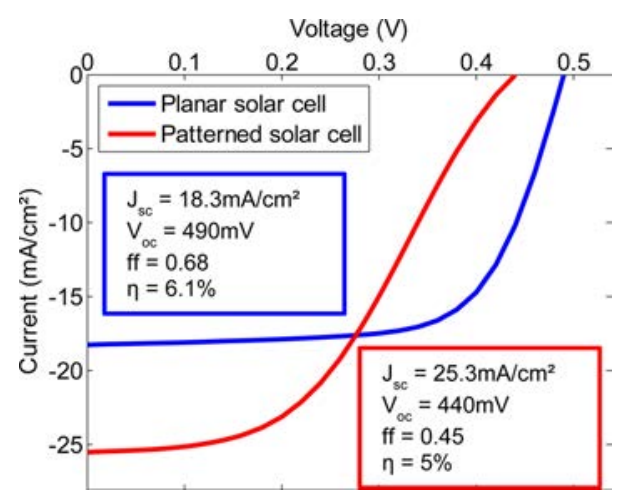

Figure 3. Current-voltage characteristics of $3 \mu \mathrm{m}$ thick planar (blue) and patterned (red) c-Si solar cells on glass under one sun illumination.

experimental results for current-voltage measurements under one sun illumination, for planar (blue curve) and patterned (red curve) solar cells. The performances achieved with planar solar cell are $J_{\text {sc }}=18.3 \mathrm{~mA} / \mathrm{cm}^{2}, V_{\text {oc }}=490 \mathrm{mV}$ and $\mathrm{ff}=0.68$. The resulting conversion efficiency of $\eta=6.1 \%$ is a remarkable efficiency for thin-film $(<5 \mu \mathrm{m}) \mathrm{c}-\mathrm{Si}$ solar cells on glass.

In the case of patterned solar cells, the short-circuit current is drastically improved, from $18.3 \mathrm{~mA} / \mathrm{cm}^{2}$ to $25.3 \mathrm{~mA} / \mathrm{cm}^{2}$. This is a $40 \%$ enhancement compared to the planar cell, despite the small material loss due to etching (8\%). However, the efficiency of this cell is limited because of a drop in the open-circuit voltage and even more importantly by the drastic decrease of the fill factor. The undesired "S-shape" of the current-voltage characteristic is likely due to an issue at an interface in the stack and is currently under investigation. Still, these results evidence an efficient light trapping in the patterned cell. In the following, we focus on the understanding of the physical phenomena that lead to this improvement of the patterned cell short-circuit current.
We performed specular reflectivity at normal incidence and external quantum efficiency (EQE) measurements on the planar and patterned cells. The results are displayed in Figure 4a. We compare these measurements with the absorption corresponding to a double pass of the light in the silicon slab. The theoretical double-pass absorption is calculated considering both a perfect antireflection coating and a perfect back mirror and using the Beer-Lambert law

$$
A(\lambda)=1-e^{-\alpha F d}
$$

where $\lambda$ is the wavelength, $\alpha$ is the wavelength-dependent absorption coefficient of silicon, $d$ is the thickness of the silicon layer, and $F$ is the light path enhancement factor. For doublepass absorption at normal incidence, $F=2$. The result is plotted together with EQE measurements in Figure 4a. The EQE of the planar solar cell presents a maximum at a wavelength of $\lambda=570$ $\mathrm{nm}$. It reaches $80 \%$, as the result of efficient antireflection effect of the ITO layer and low parasitic absorption in the TCO, spacer and passivation layers. At longer wavelengths, EQE matches double-pass absorption. Weak resonances are not perfectly resolved by the experimental setup (spectral resolution around $15 \mathrm{~nm}$ ). They originate from Fabry-Perot resonances in the slab due to nonperfect antireflection effect at the front surface of the stack.

The EQE of the patterned solar cell is much higher than the EQE of the planar solar cell on the whole spectral domain. Absorption of the planar solar cell only reaches high values around $\lambda=570 \mathrm{~nm}$ (see Supporting Information). The patterned solar cell EQE benefits from the improved antireflection properties of the inverted pyramid array compared to a simple ITO flat layer. If one would consider the pyramid array as a perfect antireflection layer similar to black silicon, ${ }^{38-40}$ the EQE of the patterned cell should decrease and match the calculated double-pass absorption. This is not the case, and EQE measurements significantly exceed double-pass absorption for wavelengths $\lambda>700 \mathrm{~nm}$. This effect is an evidence of a more efficient light trapping that presumably comes from light diffraction inside the $\mathrm{c}$-Si layer. The gain in short-circuit current $\Delta J_{\mathrm{sc}}$ that originates from diffraction can be estimated by integrating the area between the double-pass absorption and the patterned cell EQE (hatched gray in Figure 4a), weighted with a standard AM1.5G solar spectrum. The resulting short-circuit current is $\Delta J_{\mathrm{sc}}=4.4 \mathrm{~mA} / \mathrm{cm}^{2}$, which represents $20 \%$ of the $J_{\mathrm{sc}}$ of the patterned cell. 

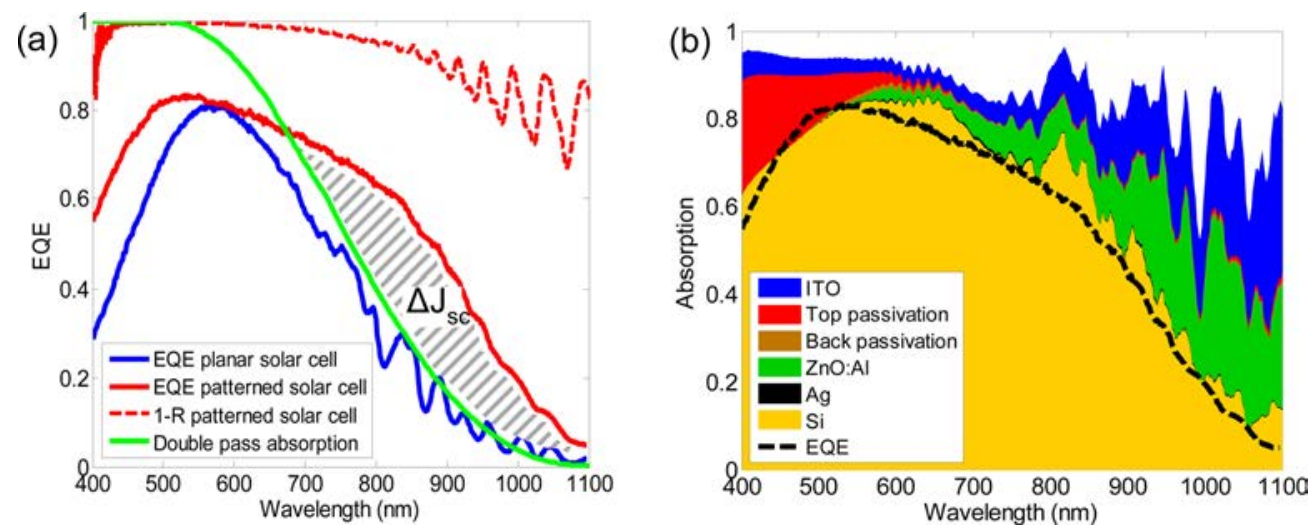

Figure 4. Spectral response of planar and patterned solar cells. (a) EQE measurements of planar and patterned solar cells, double-pass absorption calculated for a silicon slab of same thickness $d=3 \mu \mathrm{m}$, and specular reflectivity $R$ plotted as $(1-R)$. (b) Calculated absorption in each layer of patterned solar cells, from the FDTD simulation. A $10 \mathrm{~nm}$ moving average filter was applied to help the reading (see Supporting Information for the raw data). The absorption in each layer is represented by color areas: yellow for the $\mathrm{Si}$ epilayer, black for the $\mathrm{Ag}$ mirror, green for the $\mathrm{ZnO}: \mathrm{Al}$ optical spacer, brown for the back passivation layer, red for the top passivation layer, and blue for the ITO. The envelope represents the total absorption. The measured EQE of the actual device is also plotted.

(a)

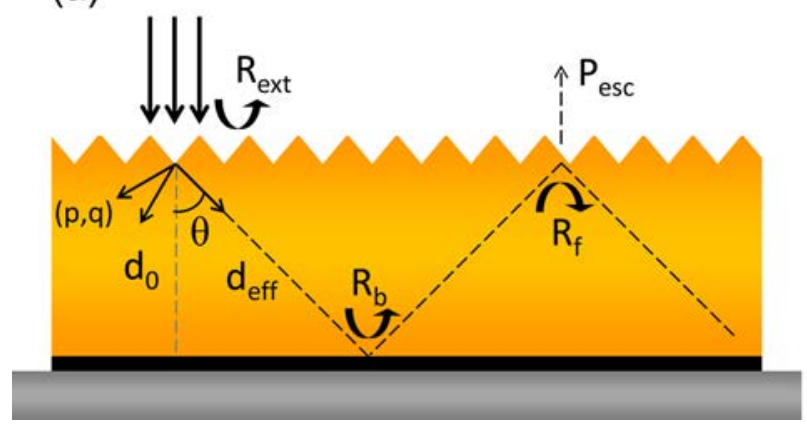

(b)

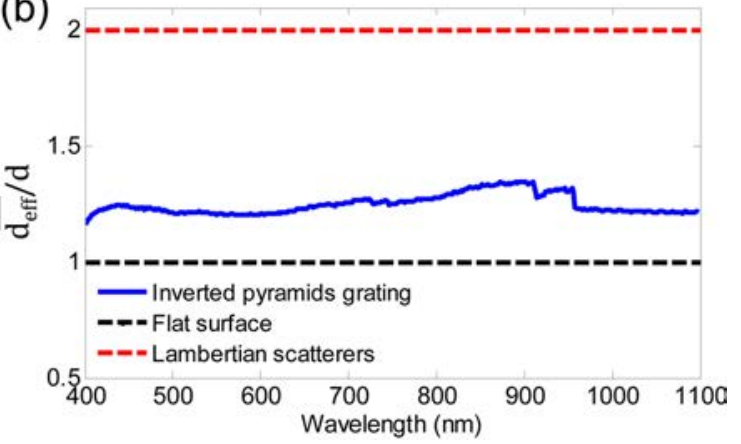

Figure 5. (a) Schematic of the simplified solar cell. (b) Normalized mean effective thickness as a function of the wavelength for various structurations of the absorber: inverted pyramid array (blue), flat surface (dashed black), and Lambertian scatterers (dashed red).

In the following, we present a thorough analysis of the optical properties of our textured c-Si solar cells. After a detailed simulation of the optical losses, we will use a simple propagation model to unveil the key parameters of the light trapping mechanism and quantify the effect of the back mirror, the increased effective thickness of diffracted light, and the photon escape probability.

First, the exact geometry of the fabricated patterned solar cell was modeled using FDTD simulation in order to understand both the absorption enhancement and the parasitic absorption losses. We calculated the distribution of absorption in the different active and nonactive layers of the patterned cell. The result is plotted in Figure $4 \mathrm{~b}$ in a cumulative form (color areas) together with the measured EQE (black dotted line). The envelope of this plot is the total absorption of the simulated stack.

Most of the absorption occurs in the electrically active silicon layer, resulting in the high $25.3 \mathrm{~mA} / \mathrm{cm}^{2}$ short-circuit current measured on our samples. The absorption in the silicon layer reaches $80 \%$ at short wavelengths, and is still close to $50 \%$ at $900 \mathrm{~nm}$. Indeed, numerical calculations and measured EQE are in very good agreement. This is an indication of high internal quantum efficiency. Assuming perfect collection of photogenerated carriers and a standard AM1.5G solar spectrum, a short-circuit current of $26.6 \mathrm{~mA} / \mathrm{cm}^{2}$ is deduced from the calculated absorption in the Si layer. However, it is clear from
Figure 4 that parasitic absorption in nonelectrically active layers is detrimental to the overall performance of the cell. On the one hand, absorption in the passivation layers is limited and corresponds to short-circuit current of $2.1 \mathrm{~mA} / \mathrm{cm}^{2}$. Moreover, carriers generated in these layers can be partly collected and contribute to the total short-circuit current of the solar cell. On the other hand, absorption in the ITO and $\mathrm{ZnO}: \mathrm{Al}$ layers is significant for $\lambda>700 \mathrm{~nm}$, and results in short-circuit current losses of $4.4 \mathrm{~mA} / \mathrm{cm}^{2}$ and $4 \mathrm{~mA} / \mathrm{cm}^{2}$, respectively. The original purpose of the $\mathrm{ZnO}: \mathrm{Al}$ back contact layer is to reduce the absorption in the Ag mirror, which is effectively the case in Figure $4 \mathrm{~b}$. However, this beneficial effect is counterbalanced by parasitic absorption. Moreover, it is likely that it increases the series resistance, hence reducing the fill factor of the cell. Overall, the optimization of both front and back TCO layers provides room for improvement and increase in $J_{\mathrm{sc}}$.

We propose to further analyze the light-trapping mechanism with a simple model based on the propagation of diffracted waves in the patterned cell. It is schematically represented in Figure 5a, where the top and back passivation and TCO layers are not shown for the sake of clarity.

The incoming photon flux can be reflected on the front surface (coefficient $R_{\text {ext }}$ ), or coupled to a set of diffracted waves of order $(p, q)$ in the silicon layer. The resulting angular distribution of photons is specific to the inverted pyramid array. It depends on the diffracted angles $\theta^{(p, q)}$ and the diffracted 
efficiencies $\mathrm{DE}^{(p, q)} \cdot \theta^{(p, q)}$ is defined as the angle between the diffracted wave $(p, q)$ and the direction normal to the plane. The diffracted efficiencies $\mathrm{DE}^{(p, q)}$ are proportional to the energy (or number of photons) in each diffracted wave. ${ }^{41}$ For the sake of simplicity, they are normalized so that the sum of the diffraction efficiencies of propagating waves at a given wavelength is 1 . They are determined numerically by FDTD simulations.

During propagation in the cell, photons can be reflected either on the back mirror (coefficient $R_{\mathrm{b}}$ ) or on the front interface (coefficient $R_{f}$ ). Both coefficients are averaged values for the angular distribution of photons, and $P_{\mathrm{esc}}=1-R_{\mathrm{f}}$ is the average probability that photons reaching the front interface escape the cell. In the following, we assume perfect antireflection effect for incoming photons $\left(R_{\text {ext }}=0\right)$, and perfect reflection on the back mirror $\left(R_{\mathrm{b}}=1\right)$. Then, the optical path enhancement $F$ induced by the nanopyramid array can have two origins: an increased effective thickness due to a mean diffraction angle $\theta>0$, and light trapping in the absorber due to reflection on the front interface $R_{\mathrm{f}}>0$. We first investigate the effect of the diffraction angles.

For a round trip propagation at angle $\theta$, the path length is $2 d_{\text {eff, }}$ where the effective thickness $d_{\text {eff }}(\theta)=d / \cos (\theta)$. Lambertian scattering is a reference case, and corresponds to a uniform occupation of the density of optical states (DOS) in the absorber. ${ }^{3}$ Its angular distribution $f_{l}(\theta)=2 \cos (\theta) \sin (\theta)$ results in a mean effective thickness $\bar{d}_{\text {eff }}=2 \int_{0}^{\pi / 2} d_{\text {eff }}(\theta)$ cos$(\theta) \sin (\theta) \mathrm{d} \theta=2 d$. In the case of inverted nanopyramid arrays, the mean effective thickness $\bar{d}_{\text {eff }}$ can be calculated as the sum the effective thicknesses of propagating diffracted orders weighted by their diffraction efficiency

$$
\overline{d_{\mathrm{eff}}}=\sum_{(p, q)} d_{\mathrm{eff}}\left(\theta^{(p, q)}\right) \times D E^{(p, q)}
$$

The result is plotted in Figure 5b, and compared to the flat interface and Lambertian cases. Most of the incoming photons are diffracted by the nanopyramid array between $\theta=33^{\circ}$ and $\theta$ $=42^{\circ}$, with a weak wavelength dependence (see Supporting Information). As a result, the normalized effective thickness $\bar{d}_{\text {eff }} / d$ displays very few variations on the whole wavelength range, with values between 1.2 and 1.35, and an average of 1.25.

If we assume no light trapping through reflection on the front surface $\left(\mathrm{R}_{\mathrm{f}}=0\right)$, we expect double-pass absorption according to eq 1 , with an optical path enhancement $F=2 \bar{d}_{\text {eff }} / d$ $=2 \times 1.25$ and $d=d_{\text {eq }}=2.75 \mu \mathrm{m}$. The result is shown in Figure 6 (dashed green line). The EQE measurement still largely exceeds double-pass absorption: the absorption enhancement due to the increased effective thickness is very weak and is not sufficient to explain the efficient light trapping evidenced by experimental measurements.

Reflection of upward photons at the front surface of the cell is thus a key in the light trapping mechanism. The light path enhancement factor $F$ in our patterned solar cell can be estimated by using a simple propagation model for light trapping of diffracted waves ${ }^{3,42}$ (see Supporting Information). For weakly absorbing medium (long wavelength range), the absorption efficiency is given by

$$
A(\lambda)=\frac{\alpha d}{\alpha d+\frac{1}{F}}
$$

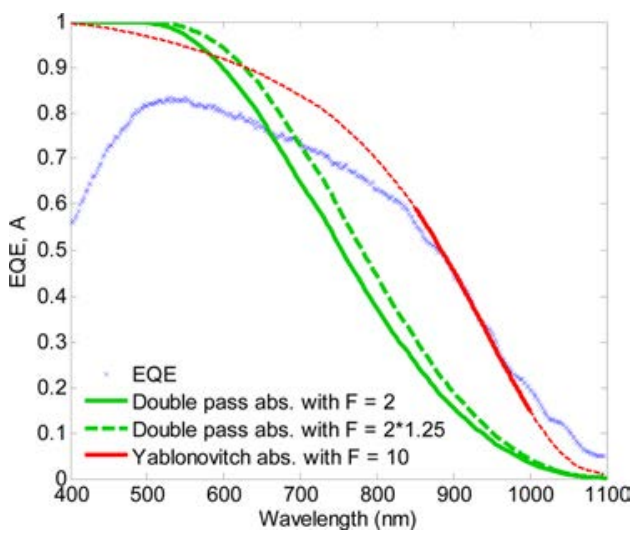

Figure 6. Comparison of the external quantum efficiency of solar cells with nanopyramid arrays with different models: double-pass absorption with $F=2$ (flat cell) and $F=2 \times 1.25$ (effective thickness induced by diffraction), and propagation model with $F=10$. The propagation model is plotted on the whole wavelength range (dotted red curve), but its domain of validity is highlighted in solid red.

As can be seen in Figure 6, this model correctly fits EQE measurements in the $850 \mathrm{~nm}-1000 \mathrm{~nm}$ wavelength range, and leads to a high enhancement factor of $F=10$.

Moreover, it can be shown that the light path enhancement factor $F$ can be simply expressed by the normalized effective thickness and the probability of escape (see Supporting Information)

$$
F=2 \frac{\overline{d_{\mathrm{eff}}}}{d} \frac{1}{P_{\mathrm{esc}}}
$$

In the case of Lambertian scattering in a medium of refractive index $n$, the probability of escape is given by $\frac{1}{P_{\text {esc }}}=n^{2}$ and leads to the Lambertian limit for light trapping $F=4 n^{2}$ ( $F \simeq 50$ for silicon). ${ }^{3}$ In the case of inverted pyramid arrays with $\bar{d}_{\text {eff }} / d=$ 1.25 and $F=10$, the probability of photon escape is estimated to $P_{\text {esc }}=0.25\left(\mathrm{R}_{\mathrm{f}}=0.75\right)$.

Overall, the light path enhancement factor $F=2 \times 1.25 \times 4$ $=10$ originates from the combined effects of the back mirror (factor of 2) and the inverted pyramid array: antireflection effect, increased effective thickness (factor of 1.25) and photon trapping in the active layer (factor of 4 ).

It is interesting to compare this wavelength-scale structuration to other common front face textures at different scales: subwavelength structuration for black silicon ${ }^{38-40}$ and conventional micron-scale pyramids. ${ }^{43}$ Black silicon acts as an effective medium with a smooth transition of the refractive index between the air and the silicon. Thus, it displays excellent antireflection properties on a large wavelength range. However, it results in no diffraction neither scattering effects, and leads to a maximum light path enhancement factor of $F=2$ if combined with a back mirror. In the case of micron-scale pyramids that can be described by geometrical optics, the refraction angle is $\theta$ $=41^{\circ}$, which is equivalent to a normalized effective thickness $d_{\text {eff }} / d=1.33$. Moreover, a large part of the rays are directly coupled out of the absorber after the first round trip. ${ }^{43}$ Hence, with an appropriate antireflective coating (ARC) and a back mirror, the maximum light path enhancement factor is $F=2 \times$ $1.33=2.66$.

We believe that the use of the propagation model to estimate the total light path enhancement factor is a convenient way to quantify the light trapping efficiency of solar cells. This 
approach is valid if the absorption of photons during a single round trip in the absorber layer is weak (here, single-pass absorption is below $15 \%$ for $\lambda>850 \mathrm{~nm}$ ). With this assumption, the angular distribution should not change significantly with the thickness, and the different parameters $\left(\bar{d}_{\text {eff }} / d, P_{\text {esc }} F\right)$ can be used to predict the performances of solar cells with the same inverted pyramid arrays and various thicknesses.

Finally, we can estimate the conversion efficiencies achievable after further optimizations. One of the main issues is to differentiate the respective role of the material quality and the surface passivation in the $V_{\text {oc }}$. With similar epitaxial silicon layers grown by low-temperature PECVD, open-circuit voltages up to $530-550 \mathrm{mV}$ and fill factors as high as 0.81 have already been demonstrated for thicknesses of $2.5-4.2 \mu \mathrm{m}^{44-46}$ Lifetime characterization (a few tens of microseconds) and device modeling have been used to analyze these performances. $^{30,47}$ They confirmed the good material quality with a low volume defect density, and concluded that these devices were likely limited by the surface defect density. Chakraborty et al. predicted an open circuit voltage close to $600 \mathrm{mV}$ with the same material quality, a thickness of $5 \mu \mathrm{m}$ and a reduced surface defect density.

It is clear that thinner solar cells are less impacted by bulk defects, but they are more sensitive to surface passivation. Branham et al. have demonstrated a $V_{\text {oc }}=593 \mathrm{mV}$ with a monocrystalline silicon solar cell of $10 \mu \mathrm{m}$ thickness. ${ }^{29}$ They concluded that their $V_{\mathrm{oc}}$ is likely limited by rear surface passivation, and provide guidelines for $V_{\text {oc }} \geq 650 \mathrm{mV} .^{29}$ Bozzola et al. and Kowalczewski et al. have also modeled the effect of surface recombination and infer a critical surface velocity to reach the ultimate efficiency determined by the bulk quality. ${ }^{7,48}$ $V_{\text {oc }}$ above $650 \mathrm{mV}$ can be achieved in the 2-5 $\mu \mathrm{m}$ thickness range with an effective surface recombination velocity of 100 $\mathrm{cm} / \mathrm{s}^{7,48}$

With a process flow similar to the one presented here, lower parasitic absorption and improved electrical performance, a patterned solar cell on glass should rapidly reach $J_{\mathrm{sc}}=30 \mathrm{~mA} /$ $\mathrm{cm}^{2}, V_{\mathrm{oc}}=600 \mathrm{mV}, \mathrm{ff}=0.8$ and $\eta=14.4 \%$. Considering new light trapping designs, such as a combined front and back structuration, ${ }^{49,50}$ the short-circuit current can reach $J_{\text {sc }}=35$ $\mathrm{mA} / \mathrm{cm}^{2}$. Thus, ultrathin $(2$ to $5 \mu \mathrm{m}) \mathrm{c}-\mathrm{Si}$ solar cells featuring the above-mentioned $\mathrm{ff}=0.8, J_{\mathrm{sc}}=35 \mathrm{~mA} / \mathrm{cm}^{2}$ and $V_{\mathrm{oc}}=600$ $\mathrm{mV}$ should result in an overall efficiency of $\eta=16.8 \%$. Our fabrication process provides a straightforward way to implement efficient passivation layers on both the front and the back surfaces. This is a mandatory step to reach $V_{\mathrm{oc}}=650 \mathrm{mV}$ and a potential efficiency of $\eta=18.2 \%$.

In conclusion, we experimentally and theoretically investigated the properties of ultrathin c-Si solar cells on glass fabricated by low cost, low temperature and scalable processes. In the case of planar solar cells with a thickness of only $3 \mu \mathrm{m}$, we demonstrated a conversion efficiency of $6.1 \%\left(J_{\text {sc }}=18.3\right.$ $\mathrm{mA} / \mathrm{cm}^{2}$ ) for ultrathin $\mathrm{c}-\mathrm{Si}$ solar cells on glass. We experimentally demonstrated short-circuit current density enhancement of $40 \%$, up to $25.3 \mathrm{~mA} / \mathrm{cm}^{2}$, using an inverted nanopyramid array on the front side of the cells (equivalent thickness $2.75 \mu \mathrm{m}$ ). A light path enhancement factor of 10 is demonstrated. It originates from excellent antireflection at the front interface, back reflection on the silver mirror, and a strong diffraction effect leading to increased effective thickness and photon trapping. The respective weight of these mechanisms has been quantitatively determined. The high reflectivity of the back mirror and the low photon escape probability at the front interface $(25 \%)$ play a key role in the efficient light trapping process.

\section{ASSOCIATED CONTENT}

\section{S Supporting Information}

The Supporting Information is available free of charge on the ACS Publications website at DOI: 10.1021/acs.nanolett.6b01240.

Supporting Information provides refractive indices ( $n$ and $k$ ) used for FDTD calculations, additional data from measurements and numerical calculations, detailed numerical analysis of the diffraction efficiencies and angular photon distribution, derivation of the analytical model for the light trapping of diffracted waves and the expression of the optical path enhancement factor $F$, and measurement results of every patterned solar cells from the same sample. (PDF)

\section{AUTHOR INFORMATION}

\section{Corresponding Author}

*E-mail: stephane.collin@lpn.cnrs.fr.

\section{Notes}

The authors declare no competing financial interest.

\section{ACKNOWLEDGMENTS}

This work was partly supported by the French ANR project NATHISOL (ANR-12-PRGE-0004) and by the RENATECH network of large nanofabrication facilities.

\section{REFERENCES}

(1) Masuko, K.; Shigematsu, M.; Hashiguchi, T.; Fujishima, D.; Kai, M.; Yoshimura, N.; Yamaguchi, T.; Ichihashi, Y.; Mishima, T.; Matsubara, N.; Yamanishi, T.; Takahama, T.; Taguchi, M.; Maruyama, E.; Okamoto, S. IEEE J. of Photovoltaics 2014, 4, 1433-1435.

(2) Richter, A.; Hermle, M.; Glunz, S. W. IEEE J. of Photovoltaics 2013, 3, 1184-1191.

(3) Yablonovitch, E. J. Opt. Soc. Am. 1982, 72, 899-907.

(4) Green, M.; Basore, P.; Chang, N.; Clugston, D.; Egan, R.; Evans, R.; Hogg, D.; Jarnason, S.; Keevers, M.; Lasswell, P.; O’Sullivan, J.; Schubert, U.; Turner, A.; Wenham, S.; Young, T. Sol. Energy 2004, 77, $857-863$.

(5) Keevers, M. J.; Young, T. L.; Schubert, U.; Green, M. A. 22nd European Photovoltaic Solar Energy Conference, Milan, September 2007.

(6) Partlin, S.; Chang, N.; Egan, R.; Young, T.; Kong, D.; Evans, R.; Clugston, D.; Lasswell, P.; Turner, A.; Dore, J.; Florian, T. 25th European Photovoltaic Solar Energy Conference and Exhibition/5th World Conference on Photovoltaic Energy Conversion 2010, 3568-3572.

(7) Bozzola, A.; Kowalczewski, P.; Andreani, L. C. J. Appl. Phys. 2014, $115,094501$.

(8) Kowalczewski, P.; Bozzola, A.; Liscidini, M.; Andreani, L. C. J. Appl. Phys. 2014, 115, 194504.

(9) Solanki, C. S.; Bilyalov, R. R.; Poortmans, J.; Nijs, J.; Mertens, R. Sol. Energy Mater. Sol. Cells 2004, 83, 101-113.

(10) Depauw, V.; Meng, X.; El Daif, O.; Gomard, G.; Lalouat, L.; Drouard, E.; Trompoukis, C.; Fave, A.; Seassal, C.; Gordon, I. IEEE J. of Photovoltaics 2014, 4, 215-223.

(11) Saha, S.; Hilali, M. M.; Onyegam, E. U.; Sarkar, D.; Jawarani, D.; Rao, R. A.; Mathew, L.; Smith, R. S.; Xu, D.; Das, U. K.; Sopori, B.; Banerjee, K. Appl. Phys. Lett. 2013, 102, 163904.

(12) Haschke, J.; Amkreutz, D.; Korte, L.; Ruske, F.; Rech, B. Sol. Energy Mater. Sol. Cells 2014, 128, 190-197.

(13) Gomard, G.; Drouard, E.; Letartre, X.; Meng, X.; Kaminski, A.; Fave, A.; Lemiti, M.; Garcia-Caurel, E.; Seassal, C. J. Appl. Phys. 2010, $108,123102$. 
(14) Chong, T. K.; Wilson, J.; Mokkapati, S.; Catchpole, K. R. J. Opt. 2012, 14, 024012 .

(15) Wang, K. X.; Yu, Z.; Liu, V.; Cui, Y.; Fan, S. Nano Lett. 2012, 12, 1616-1619.

(16) Hong, L.; Rusli; Wang, X.; Zheng, H.; Wang, H.; Xiaoyan, X.; Yu, H. J. Appl. Phys. 2014, 116, 074310.

(17) Atwater, H. A.; Polman, A. Nat. Mater. 2010, 9, 205-213.

(18) Beck, F. J.; Polman, A.; Catchpole, K. R. J. Appl. Phys. 2009, 105, 114310.

(19) Spinelli, P.; Verschuuren, M.; Polman, A. Nat. Commun. 2012, 3, 692.

(20) Pala, R. A.; Liu, J. S. Q.; Barnard, E. S.; Askarov, D.; Garnett, E. C.; Fan, S.; Brongersma, M. L. Nat. Commun. 2013, 4, 2095.

(21) Eisenlohr, J.; Tucher, N.; Höhn, O.; Hauser, H.; Peters, M.; Kiefel, P.; Goldschmidt, J. C.; Bläsi, B. Opt. Express 2015, 23, A502A518.

(22) Tucher, N.; Eisenlohr, J.; Kiefel, P.; Höhn, O.; Hauser, H.; Peters, M.; Müller, C.; Goldschmidt, J. C.; Bläsi, B. Opt. Express 2015, 23, A1720-A1734.

(23) Petermann, J. H.; Zielke, D.; Schmidt, J.; Haase, F.; Rojas, E. G.; Brendel, R. Prog. Photovoltaics 2012, 20, 1-5.

(24) Moslehi, M. M.; Kapur, P.; Kramer, J.; Rana, V.; Seutter, S.; Deshpande, A.; Stalcup, T.; Kommera, S.; Ashjaee, J.; Calcaterra, A.; Grupp, D.; Dutton, D.; Brown, R. PV Asia Pacific Conference 2012, 24. (25) Jeong, S.; McGehee, M. D.; Cui, Y. Nat. Commun. 2013, 4, 2950.

(26) Li, H.; Li, G.; Ho, J.; Wong, M.; Kwok, H. S. Proc. $29^{\text {Th }}$ European Photovoltaic Solar Energy Conference 2014, 1628-1630.

(27) Lu, Y.; Lal, A. Nano Lett. 2010, 10, 4651-4656.

(28) Sheng, X.; Broderick, L. Z.; Kimerling, L. C. Opt. Commun. 2014, 314, 41-47.

(29) Branham, M. S.; Hsu, W. C.; Yerci, S.; Loomis, J.; Boriskina, S. V.; Hoard, B. R.; Han, S. E.; Chen, G. Adv. Mater. 2015, 27, 21822188.

(30) Cariou, R.; Massiot, I.; Ruggeri, R.; Ramay, N.; Tang, J.; Cattoni, A.; Collin, S.; Nassar, J.; Roca i Cabarrocas, P. Proc. $28^{\text {Th }}$ European Photovoltaic Solar Energy Conference 2013, 2225-2227.

(31) Cariou, R.; Chen, W.; Cosme-Bolanos, I.; Maurice, J.-L.; Foldyna, M.; Depauw, V.; Patriarche, G.; Gaucher, A.; Cattoni, A.; Massiot, I.; Collin, S.; Cadel, E.; Pareige, P.; Roca i Cabarrocas, P. Progress in Photovoltaics: Research and Applications 2016, 1075 DOI: $10.1002 /$ pip.2762.

(32) Moreno, M.; Roca i Cabarrocas, P. EPJ Photovoltaics 2010, 1, 10301.

(33) Moreno, M.; Patriarche, G.; Roca i Cabarrocas, P. J. Mater. Res. 2013, 28, 1626-1632.

(34) Chen, W.; Cariou, R.; Foldyna, M.; Depauw, V.; Trompoukis, C.; Drouard, E.; Lalouat, L.; Harouri, A.; Liu, J.; Fave, A.; Orobtchouk, R.; Mandorlo, F.; Seassal, C.; Massiot, I.; Dmitriev, A.; Lee, K.-D.; Roca i Cabarrocas, P. J. Phys. D: Appl. Phys. 2016, 49, 125603.

(35) Cattoni, A.; Ghenuche, P.; Haghiri-Gosnet, A. M.; Decanini, D.; Chen, J.; Pelouard, J. L.; Collin, S. Nano Lett. 2011, 11, 3557-3563.

(36) Cattoni, A.; Faustini, M.; Yacomotti, A. M.; Decanini, D.; Grosso, D.; Haghiri-Gosnet, A. M. Unpublished.

(37) Schmitt, H.; Duempelmann, P.; Fader, R.; Rommel, M.; Bauer, A. J.; Frey, L.; Brehm, M.; Kraft, A. Microelectron. Eng. 2012, 98, 275278.

(38) Koynov, S.; Brandt, M. S.; Stutzmann, M. Appl. Phys. Lett. 2006, $88,203107$.

(39) Liu, X.; Coxon, P. R.; Peters, M.; Hoex, B.; Cole, J. M.; Fray, D. J. Energy Environ. Sci. 2014, 7, 3223-3263.

(40) Savin, H.; Repo, P.; von Gastrow, G.; Ortega, P.; Calle, E.; Garin, M.; Alcubilla, R. Nat. Nanotechnol. 2015, 10, 624-628.

(41) Moharam, M. G.; Grann, E. B.; Pommet, D. A.; Gaylord, T. K. J. Opt. Soc. Am. A 1995, 12, 1068-1076.

(42) Green, M. A. Prog. Photovoltaics 2002, 10, 235-241.

(43) Campbell, P.; Green, M. A. J. Appl. Phys. 1987, 62, 243-249.

(44) Cariou, R.; Labrune, M.; Roca i Cabarrocas, P. Sol. Energy Mater. Sol. Cells 2011, 95, 2260-2263.
(45) Cariou, R.; Ruggeri, R.; Chatterjee, P.; Gentner, J.-L.; Roca i Cabarrocas, P. Proc. SPIE 2012, 8470, 84700B.

(46) Bruneau, B.; Cariou, R.; Dornstetter, J. C.; Lepecq, M.; Maurice, J. L.; Roca i Cabarrocas, P.; Johnson, E. V. IEEE J. of Photovoltaics 2014, 4, 1361-1367.

(47) Chakraborty, S.; Cariou, R.; Labrune, M.; Roca i Cabarrocas, P.; Chatterjee, P. EPJ Photovoltaics 2013, 4, 45103.

(48) Kowalczewski, P.; Redorici, L.; Bozzola, A.; Andreani, L. C. J. Opt. 2016, 18, 054001.

(49) Meng, X.; Drouard, E.; Gomard, G.; Peretti, R.; Fave, A.; Seassal, C. Opt. Express 2012, 20, A560-A571.

(50) Wang, K. X.; Yu, Z.; Liu, V.; Cui, Y.; Fan, S. Nano Lett. 2012, 12, $1616-1619$. 